\title{
Artemesia annua extract prevents glyoxal-induced cell injury in retinal microvascular endothelial cells during glaucoma
}

\author{
Shun Jiang, Qin Wang, Jiawen Ling* \\ Department of Ophthalmology, Zhangjiagang Third People's Hospital, Zhangjiagang City, Jiangsu 215600, China \\ *For correspondence: Email: LuanneOhiji@yahoo.com; Tel: +865125844 1983
}

Sent for review: 30 October 2017

Revised accepted: 22 January 2018

\begin{abstract}
Purpose: To investigate the effect of Artemesia annua extract on glyoxal-induced injury in retinal microvascular endothelial cells (HRECs).

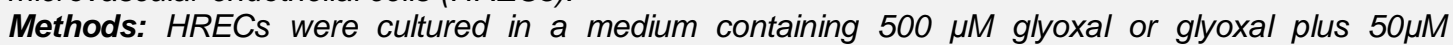
Artemesia annua extract, or in the medium alone for $24 \mathrm{~h}$. Apoptosis was analysed by flow cytometry using annexin $V$ and propidium iodide staining. Changes in mitochondrial membrane potential were determined by $\mathrm{JC}$-1 staining.

Results: When HRECs were cultured in a medium of $500 \mu \mathrm{M}$ glyoxal, a significant $(p<0.05)$ decrease in caspase-3 expression was observed. However, treatment of HRECs with Artemesia annua extract $(50 \mu \mathrm{M})$ inhibited the glyoxal-mediated decrease in caspase-3 expression. The extract also inhibited caspase-3 proteolysis, as was evident from the reduction in the level of cleaved caspase-3. Upregulation of ROS production by glyoxal in HRECs was inhibited by treatment with the extract. The viability of HRECs was significantly decreased by glyoxal $(p<0.05)$, but the decrease in viability was significantly reversed by Artemesia annua extract $(p<0.05)$. The extract also reduced gyloxal-induced apoptosis in HRECs from 17.3 to $2.6 \%(p<0.001)$. Results from JC-1 staining showed significantly $(p<$ 0.001 ) higher level of green fluorescence in HRECs cultured with glyoxal. However, the glyoxal-induced increase in green fluorescence level was significantly $(p<0.01)$ reduced on exposure to Artemesia annua extract.

Conclusion: Artemesia annua extract prevents oxidative damage to HRECs via inhibition of ROS production, up-regulation of caspase-3 expression and suppression of caspase-3 proteolysis. Therefore, Artemesia annua can potentially be used for the development of a new drug for the prevention of retinal injury in glaucoma.
\end{abstract}

Keywords: Artemesia annua, Retinal injury, Glaucoma, Green fluorescence, cleaved caspase-3, ROS production

\begin{abstract}
This is an Open Access article that uses a funding model which does not charge readers or their institutions for access and distributed under the terms of the Creative Commons Attribution License (http://creativecommons.org/licenses/by/4.0) and the Budapest Open Access Initiative (http://www.budapestopenaccessinitiative.org/read), which permit unrestricted use, distribution, and reproduction in any medium, provided the original work is properly credited.

Tropical Journal of Pharmaceutical Research is indexed by Science Citation Index (SciSearch), Scopus, International Pharmaceutical Abstract, Chemical Abstracts, Embase, Index Copernicus, EBSCO, African Index Medicus, JournalSeek, Journal Citation Reports/Science Edition, Directory of Open Access Journals (DOAJ), African Journal Online, Bioline International, Open-J-Gate and Pharmacy Abstracts
\end{abstract}

\section{INTRODUCTION}

Diabetic retinopathy is a common cause of vision loss in people with diabetes mellitus throughout the world [1]. It causes cellular degradation of retina, and is characterised by hyperglycaemia [1]. The level of glyoxal, which is a toxic chemical, increases during hyperglycaemia, 
leading to oxidative damage through upregulation of generation of reactive oxygen species (ROS) [2,3]. In hyperglycaemic patients, the development of diabetic retinopathy is facilitated by ROS production through increased capillary permeability [4-6]. The high level of blood glucose promotes O-linked $\beta$-N-acetyl glucosamine expression which plays a crucial role in the development of diabetes $[7,8]$.

High intraocular pressure is considered a major pre-disposing factor for glaucoma, and it leads to optic nerve shrinkage and retinal ganglion cell apoptosis [9-11]. This disorder cannot be remedied even after transplantation of stem cells or ganglion cells $[9,12]$. Current treatments for glaucoma focus mainly on neuro-protection. Identification of novel and efficient neuroprotective agents is of immense importance for the prevention of retinal injury during glaucoma. Studies have been carried out to investigate the protective effects of various compounds against retinal injury during glaucoma in animal models. The extracts which have been investigated in animal models for this purpose include Lycium barbarum Lynn and Salvia miltiorrhiza [13-16].

In the present study, the effect of Artemesia annua extract on the viability of HRECs was investigated. Glyoxal was added to HRECs cultures to induce oxidative stress, and the protective effect of Artemesia annua extract was investigated.

\section{EXPERIMENTAL}

\section{Culturing of HRECs}

The HRECs were obtained from Cell Systems Corp. (CSC; Kirkland, WA, USA) and expression of ROS was analysed by immunofluorescence staining. The cells were grown in DEM medium containing $5 \%$ FBS and $1 \%$ penicillin and streptomycin in an atmosphere of $5 \% \mathrm{CO}_{2}$ at a temperature of $37^{\circ} \mathrm{C}$.

\section{Western blot assay}

The HRECs were cultured in a medium containing $500 \mu \mathrm{M}$ glyoxal alone (control) or glyoxal and Artemesia annua extract $(50 \mu \mathrm{M})$ for $24 \mathrm{~h}$, and washed twice with phosphate-buffered saline (PBS). The cells were then treated with lysis buffer supplemented with protease inhibitor cocktail. The proteins from the cell extract were separated by subjecting equal amounts $(30 \mu \mathrm{g})$ to fractionation on $10 \%$ polyacrylamide gel. The separated proteins were subsequently transferred at $200 \mathrm{~mA}$ to polyvinylidene fluoride membranes for $2 \mathrm{~h}$. The membranes were blocked by incubation with $0.05 \%$ non-fat milk powder in TBST for $2 \mathrm{~h}$ at $37^{\circ} \mathrm{C}$. After blocking, the membranes were incubated with primary antibodies overnight at $4{ }^{\circ} \mathrm{C}$.

The antibodies used were rabbit anti-caspase-3 (catalog no. 9665; dilution 1: 1, 000) and anti- $\beta$ actin (catalog no. 8457; dilution 1:1,000; both from Cell Signalling Technology; Danvers, MA, USA). After incubation, the blots were washed twice with PBS-T (0.1\% Tween-20 in PBS), and then incubated with goat horseradish peroxidaseconjugated anti-mouse IgG (dilution 1: 5, 000; Pierce Biotechnology, Rockford, IL, USA) secondary antibody at room temperature for $1 \mathrm{~h}$. The blots were thereafter visualized under Odyssey (LI-COR Biosciences, Lincoln, NE, USA).

\section{Analysis of ROS production}

Analysis of ROS production in HRECs was carried out using DCFH-DA. Into the cell cultures, $5 \mu \mathrm{M}$ of DCFH-DA was added and the cells were cultured for $30 \mathrm{~min}$, and then lysed using sodium hydroxide $(400 \mathrm{mM})$ for $2 \mathrm{~h}$ at room temperature. The lysates were used for determination of ROS production by measuring the intensity of fluorescence at absorption and emission wavelengths of 480 and $430 \mathrm{~nm}$, respectively in a multi-well plate reader (Synergy 2, BioTek Instruments Inc., Winooski, VT, USA). Bicinchoninic acid (BCA) protein assay kits (Pierce Biotechnology) were used for protein determination. Quantification was performed as the ratio of the intensity of fluorescence to concentration of protein.

\section{Evaluation of cell viability}

The HRECs were seeded into 96-well culture plates at a density of $1 \times 10^{4}$ cells per well and cultured for $24 \mathrm{~h}$ under an atmosphere of $5 \%$ $\mathrm{CO}_{2}$ at $37{ }^{\circ} \mathrm{C}$. The cells were then assigned to three groups: one group was cultured in a medium containing $500 \mu \mathrm{M}$ glyoxal; the second group was cultured in a medium containing 500 $\mu \mathrm{M}$ glyoxal and $50 \mu \mathrm{M}$ Artemesia annua extract, while the third group (normal control) was cultured in medium alone. After $24 \mathrm{~h}, \mathrm{CCK}-8$ solution $(10 \mu \mathrm{L})$ was added to the plates and incubation was continued further for $4 \mathrm{~h}$.

At the end of incubation, the absorbance for each plate was recorded three times in a multi-well plate reader at $450 \mathrm{~nm}$. The effect of hydrogen peroxide on HRECs viability was analysed on incubation with $200 \mu \mathrm{M} \mathrm{H} \mathrm{H}_{2} \mathrm{O}_{2}$ in the medium containing $5 \%$ of FBS. 


\section{Flow cytometry analysis}

The cells were cultured in a medium containing $500 \mu \mathrm{M}$ glyoxal or glyoxal $(500 \mu \mathrm{M})$ plus Artemesia annua extract $(50 \mu \mathrm{M})$, or medium alone, for $24 \mathrm{~h}$. After incubation, the cell pellet was re-suspended in binding buffer, and incubated with $5 \mathrm{ml}$ of Annexin $\mathrm{V}$ (conjugated with FITC; BD Biosciences) and $10 \mathrm{ml}$ of PI (BD Biosciences), in the dark for $5 \mathrm{~min}$. Finally, the cells were examined in a FACScan flow cytometer (Becton-Dickinson, Franklin Lakes, NJ, USA).

\section{Determination of mitochondrial membrane potential}

In the analysis of changes in mitochondrial membrane potential, JC-1 stain was used. The three groups of cells cultured in different media as outlined earlier, were analysed for changes in membrane potential. The cells were stained with $\mathrm{JC}-1 \quad(2.5 \mu \mathrm{M})$ for $20 \mathrm{~min}$ at $37{ }^{\circ} \mathrm{C}$, and subsequently rinsed thrice in PBS. Images were captured at $525 \mathrm{~nm}$ and $557 \mathrm{~nm}$ absorption and emission wavelengths, respectively, using an inverted fluorescence microscope (Leica Microsystems). The intensity of fluorescence was analysed using Image-Pro Plus v6.0 software.

\section{Statistical analysis}

The experimental data are expressed as mean \pm standard deviation (SD). Statistical analysis was performed with SPSS 13.0 statistical software (SPSS, Inc. Chicago, IL, USA). One-way analysis of variance (ANOVA) was used, followed by Dunnett's test for multiple comparisons. $P<0.05$ was considered to indicate statistically significant difference.

\section{RESULTS}

\section{Effect of Artemisia annua on expression of caspase-3 in HPRECs}

Glyoxal caused a significant decrease in the expression of caspase-3 in HRECs. However, treatment of the glyoxal-exposed HRECs with Artemesia annua extract led to a significant $(p<$ 0.01 ) increase in caspase- 3 expression (Figure 1). Proteolysis of caspase- 3 was inhibited by Artemesia annua extract, as was evident in reduction of the level of cleaved caspase-3. These findings suggest that Artemesia annua extract protected HRECs from glyoxal-induced damage.

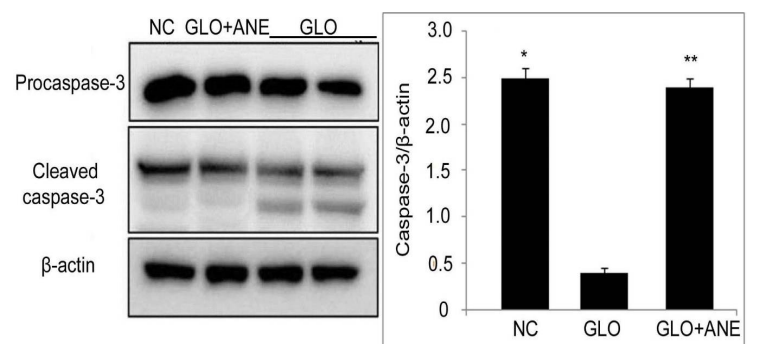

Figure 1: Artemesia annua extract inhibited glyoxalinduced cleavage of caspase-3 in HRECs. The quantification of caspase-3 level in HRECs following treatment with glyoxal and/or Artemesia annua extract was performed using Quantity One software. ${ }^{*} p<0.05$ (glyoxal vs. normal control); ${ }^{* *} p<0.01$ (glyoxal vs. glyoxal + Artemesia annua extract)

\section{Artemesia annua extract inhibited production of ROS in glyoxal-treated HRECs}

Examination using fluorescence microscopy showed higher intensity of bright spots in HRECs treated with glyoxal, when compared to untreated HRECs (Figure 2). The ROS production induced by glyoxal in HRECs was inhibited by treatment with Artemesia annua extract $(p<0.05)$. Therefore, the extract protected HRECs from glyoxal-mediated oxidative damage.

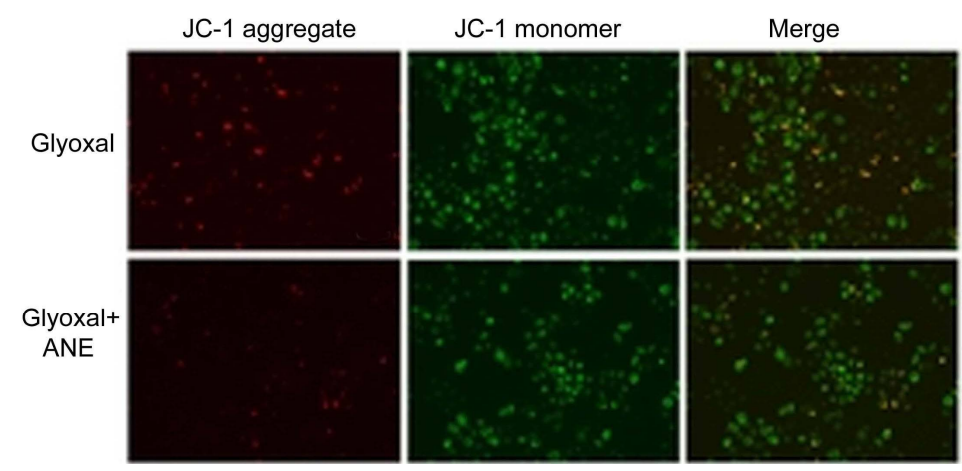

Figure 2: Artemesia annua extract blocked up-regulation of ROS production by glyoxal. HRECs, after treatment with glyoxal and/or Artemesia annua extract, were analysed for ROS content by fluorescence microscopy using DCFH-DA staining. An automatic microplate reader was used for quantitative analysis of changes in ROS

Trop J Pharm Res, February 2018; 17(2): 247 


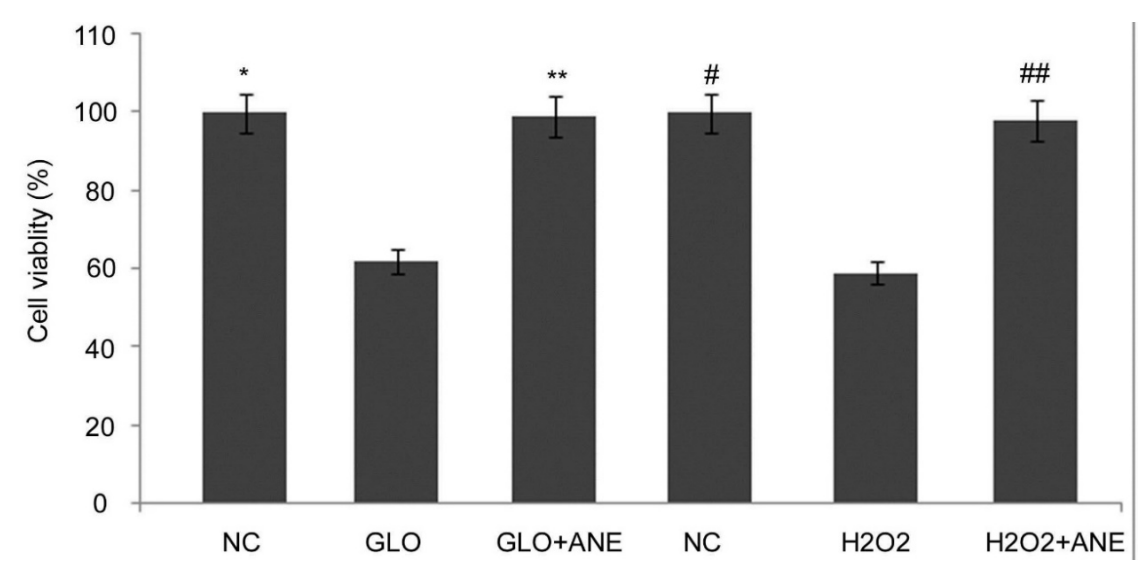

Figure 3: Artemesia annua extract prevented glyoxal-mediated loss of viability in HRECs. ${ }^{*} p<0.005$ (glyoxal vs. control); ${ }^{* *} p>0.05$ (glyoxal vs glyoxal + Artemesia annua extract). HRECs were exposed to $\mathrm{H}_{2} \mathrm{O}_{2}$ and then treated with Artemesia annua extract for 24 h. ${ }^{\#} p<0.001\left(\mathrm{H}_{2} \mathrm{O}_{2}\right.$ vs. normal control); $\# p<0.0001\left(\mathrm{H}_{2} \mathrm{O}_{2}\right.$ vs $\mathrm{H}_{2} \mathrm{O}_{2}+$ Artemesia annua extract $)$
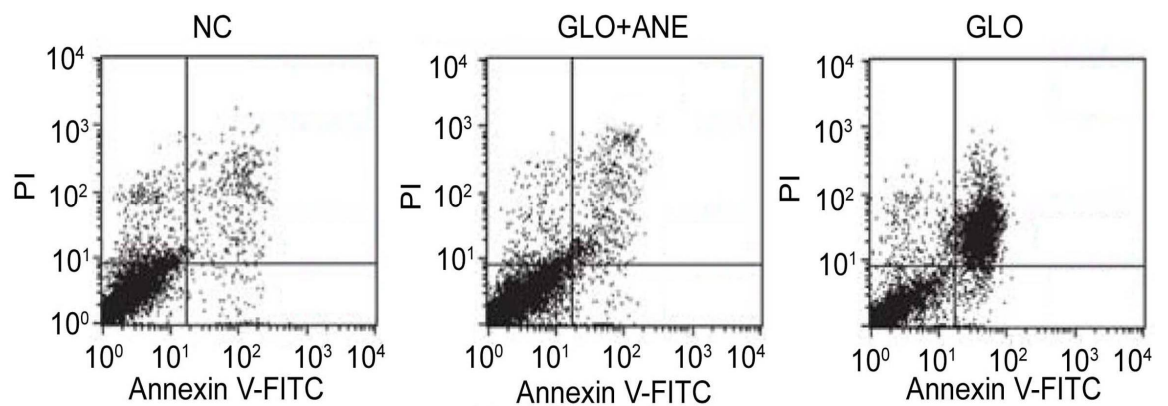

Figure 4: Artemesia annua extract prevented glyoxal-induced apoptosis in HRECs. Flow cytometry was used to determine the effect of glyoxal and Artemesia annua extract on apoptosis in HRECs after $24 \mathrm{~h}$. Staining of HRECs was performed using Annexin V-FITC and PI, followed by FACS Artemesia annua extract prevented glyoxal-
mediated decrease in viability of HRECs

Changes in viability of HRECs treated with Artemesia annua extract were analysed after $24 \mathrm{~h}$. Glyoxal treatment led to a significant $(p<$ $0.005)$ decrease in HRECs viability, when compared to the untreated control (Figure 3). However, after Artemesia annua extract treatment, the viability of HRECs exposed to glyoxal was significantly increased. In order to confirm if Artemesia annua extract prevented inhibition of viability through attenuation of oxidative damage, HRECs were treated with $\mathrm{H}_{2} \mathrm{O}_{2}(200 \mu \mathrm{M})$ for $2 \mathrm{~h}$. The results showed that $\mathrm{H}_{2} \mathrm{O}_{2}$ reduced HRECs viability, but the inhibitory effect was reversed by Artemesia annua extract (Figure 3).

\section{Artemesia annua extract blocked apoptosis in HRECs}

Glyoxal treatment increased the percentage of apoptosis in HRECs to $17.3 \%$, when compared to $2.5 \%$ in the untreated control (Figure 4). However, addition of Artemesia annua extract to the glyoxal-treated cultures of HRECs led to significant reduction of apoptosis to $2.6 \%$ (Figure 4). Thus, it is clear that Artemesia annua extract prevented glyoxal-mediated increase in the percentage of apoptosis in HRECs.

\section{Artemesia annua extract prevented glyoxal- mediated alteration in mitochondrial membrane potential}

The results from JC-1 probe showed a significantly higher level of green fluorescence in HRECs cultured with glyoxal (Figure 5). However, the level of green fluorescence was significantly reduced in glyoxal-treated HRECs on exposure to Artemesia annua extract (Figure 5). These observations suggest that Artemesia annua extract blocked changes in mitochondrial membrane potential caused by glyoxal.

\section{DISCUSSION}

Reactive oxygen species (ROS) are responsible for cellular stress that results in retinal injury and loss of vision $[5,17,18]$. Increased blood glucose levels for prolonged durations at the initial stage 


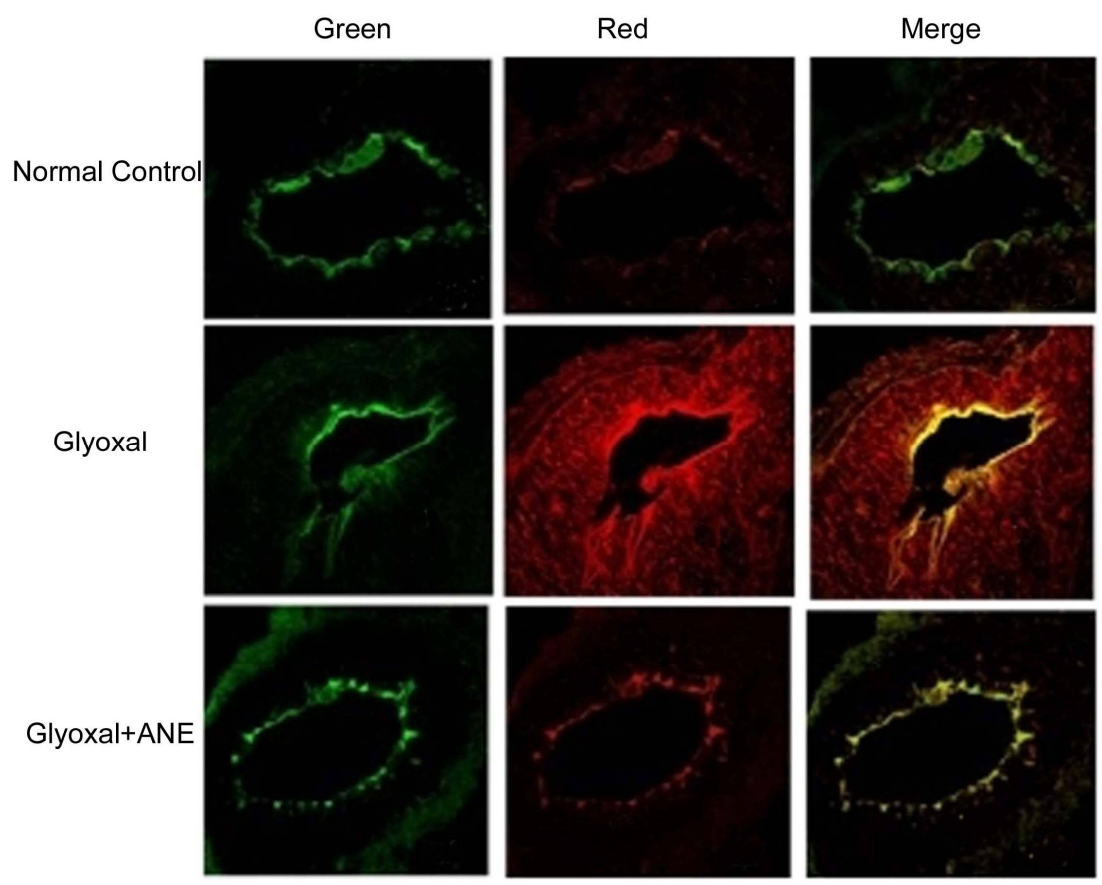

Figure 5: Effect of Artemesia annua extract on glyoxal-induced changes in mitochondrial membrane potential in HRECs. The cells were incubated with JC-1 after treatment with glyoxal and Artemesia annua extract for $24 \mathrm{~h}$. Inverted fluorescent microscope and Image-Pro Plus software were used for measurement of fluorescence. The loss in membrane potential in cells treated with glyoxal was prevented by addition of Artemesia annua extract

of diabetic retinopathy result in generation of ROS and the development of retinal hypoxia [19]. Studies have shown that following surgical eye treatment in patients with proliferative diabetic retinopathy, the expression of hypoxia-inducible factor (HIF)-1a is high in vitreous fluid [20]. Upregulation of HIF-1a expression is a characteristic feature of hypoxia and onset of oxidative damage [21]. Exposure of HRECs to glyoxal leads to marked increases in the rate of apoptosis [22]. It has been reported that the suppression of ROS production inhibits apoptosis and increases the viability of HRECs [22]. Indeed, several studies have shown that the production of ROS plays a vital role in the development of retinal injury and diabetic retinopathy [23-26].

The results of the present study showed that Artemesia annua extract inhibited glyoxalinduced ROS generation in HRECs. Apoptosis in endothelial cells which form lining of the capillary wall plays a vital role in the onset of diabetic retinopathy. The present study has demonstrated that glyoxal-mediated increase in apoptosis in HRECs was significantly inhibited by Artemesia annua extract. In order to understand the mechanism of Artemesia annua extract-mediated blockage of glyoxal-induced apoptosis in HRECs, caspase-3 and mitochondrial membrane potential were determined. Increases in the level of cleaved caspase-3 enhance the rate of apoptosis in HRECs. Caspase-3 is activated by proteolysis into cleaved caspase- 3 which has an important role in inducing apoptosis [27-29]. Glyoxal treatment has been shown to increase the level of cleaved caspase-3 level and decrease overall caspase-3 activity in HRECs after $24 \mathrm{~h}[28,29]$. In the current study, the addition of Artemesia annua extract to the glyoxal-treated cultures of HRECs led to increased level of caspase-3. In presence of glyoxal, treatment of HRECs with Artemesia annua extract prevented reduction in viability. In order to confirm the anti-oxidative mode of action of Artemesia annua extract in blocking glyoxalmediated loss of viability, HRECs were treated with $\mathrm{H}_{2} \mathrm{O}_{2}$. The results revealed that the $\mathrm{H}_{2} \mathrm{O}_{2}$. induced reduction in HREC viability was inhibited by the extract. Thus, Artemesia annua extract acted as a therapeutic agent for prevention of apoptosis in HRECs through a mechanism involving targeting of ROS production.

Mitochondria play important roles in the protection of cellular damage by inhibiting ROS production, and regulating calcium ion channels $[30,31]$. In the present study, results from JC-1 staining and inverted fluorescent microscopy showed that Artemesia annua extract prevented changes in mitochondria membrane potential induced by oxidative damages in HRECs.

\section{CONCLUSION}

The findings of the present study provide indicate

Trop J Pharm Res, February 2018; 17(2): 249 
that Artemesia annua extract prevents glyoxalinduced apoptosis in HREC by inhibition of ROS production and blockage of caspase-3 cleavage. The extract also prevents changes in mitochondrial membrane potential. Thus, Artemesia annua has potentials to be developed into a new drug for the treatment of diabetic retinopathy.

\section{DECLARATIONS}

\section{Conflict of Interest}

No conflict of interest associated with this work.

\section{Contribution of Authors}

The authors declare that this work was done by the authors named in this article and all liabilities pertaining to claims relating to the content of this article will be borne by them.

Shun Jiang drafted the manuscript and did all the experiments under the supervision of Jiawen Ling. Qin Wang collected materials and did statistical analysis.

\section{REFERENCES}

1. Cheung $N$, Mitchell $P$, Wong $T Y$. Diabetic retinopathy. Lancet 2010; 376: 124-136.

2. Shangari N, Bruce WR, Poon R, O'Brien PJ. Toxicity of glyoxals - role of oxidative stress, metabolic detoxification and thiamine deficiency. Biochem Soc Trans 2003; 31: 1390-1393.

3. Li SY, Sigmon VK, Babcock SA, Ren J. Advanced glycation endproduct induces ROS accumulation, apoptosis, MAP kinase activation and nuclear $O$ GlcNAcylation in human cardiac myocytes. Life Sci 2007; 80: 1051-1056.

4. Mohamed IN, Soliman SA, Alhusban A, Matragoon $S$, Pillai BA, Elmarkaby $A A$, El-Remessy $A B$. Diabetes exacerbates retinal oxidative stress, inflammation, and microvascular degeneration in spontaneously hypertensive rats. Mol Vis 2012; 18: 1457-1466.

5. Santos JM, Mohammad G, Zhong Q, Kowluru RA. Diabetic retinopathy, superoxide damage and antioxidants. Curr Pharm Biotechnol 2011; 12: 352-361.

6. Fernandes $R$, Hosoya $K$, Pereira $P$. Reactive oxygen species down regulate glucose transport system in retinal endothelial cells. Am J Physiol Cell Physiol 2011; 300: C927-C936.

7. Gurel Z, Sieg KM, Shallow KD, Sorenson CM, Sheibani $N$. Retinal O-linked $\mathrm{N}$-acetylglucosamine protein modifications: implications for postnatal retinal vascularization and the pathogenesis of diabetic retinopathy. Mol Vis 2013; 19: 1047-1059.
8. Xu C, Liu G, Liu X, Wang F. O-GlcNAcylation under hypoxic conditions and its effects on the blood-retinal barrier in diabetic retinopathy. Int $\mathrm{J} \mathrm{Mol} \mathrm{Med} \mathrm{2014;} \mathrm{33:}$ 624-632.

9. Worley A, Grimmer-Somers K. Risk factors for glaucoma: what do they really mean? Aust J Prim Health 2011; 17: 233-239.

10. Wong VH, Bui BV, Vingrys AJ. Clinical and experimental links between diabetes and glaucoma. Clin Exp Optom 2011; 94: 4-23.

11. Yanagi M, Kawasaki R, Wang JJ, Wong TY, Crowston J, Kiuchi $Y$. Vascular risk factors in glaucoma: a review. Clin Experiment Ophthalmol 2011; 39: 252-258.

12. Hayreh SS. Neovascular glaucoma. Prog Retin Eye Res 2007; 26: 470-485.

13. Chan HC, Chang RC, Koon-Ching Ip A, Chiu K, Yuen $W H$, Zee SY, So KF. Neuroprotective effects of Lycium barbarum Lynn on protecting retinal ganglion cells in an ocular hypertension model of glaucoma. Exp Neurol 2007; 203: 269-273.

14. Mi XS, Zhong JX, Chang RC, So KF. Research advances on the usage of traditional Chinese medicine for neuroprotection in glaucoma. J Integr Med 2013; 11: 233-240.

15. Cheng TO. Cardiovascular effects of Danshen. Int $J$ Cardiol 2007; 121: 9-22.

16. Qin RA, Yao XX, Huang ZY. Effects of compound danshen tablets on spatial cognition and expression of brain beta-amyloid precursor protein in a rat model of Alzheimer's disease. J Tradit Chin Med 2012; 32: 63-66.

17. Sun $J, X u Y$, Sun $S$, Sun $Y$, Wang $X$. Intermittent high glucose enhances cell proliferation and VEGF expression in retinal endothelial cells: the role of mitochondrial reactive oxygen species. Mol Cell Biochem 2010; 343: 27-35.

18. Al-Shabrawey $M$, Smith $S$. Prediction of diabetic retinopathy: role of oxidative stress and relevance of apoptotic biomarkers. EPMA J 2010; 1: 56-72.

19. Kennedy $A$, Frank RN. The influence of glucose concentration and hypoxia on VEGF secretion by cultured retinal cells. Curr Eye Res 2011; 36: 168-177.

20. Loukovaara S, Koivunen P, Inglés M, Escobar J, Vento $M$, Andersson S. Elevated protein carbonyl and HIF-1a levels in eyes with proliferative diabetic retinopathy. Acta Ophthalmol 2014; 92: 323-327.

21. Izuta $H$, Matsunaga $N$, Shimazawa $M$, Sugiyama $T$, Ikeda $T$, Hara $H$. Proliferative diabetic retinopathy and relations among antioxidant activity, oxidative stress, and VEGF in the vitreous body. Mol Vis 2010; 16: 130136.

22. Moley KH, Mueckler MM. Glucose transport and apoptosis. Apoptosis 2000; 5: 99-105.

23. Kowluru RA, Chan PS. Oxidative stress and diabetic retinopathy. Exp Diabetes Res 2007: 43603.

24. Brownlee M. The pathobiology of diabetic complications: a unifying mechanism. Diabetes 2005; 54: 1615-1625.

25. Caldwell RB, Bartoli M, Behzadian MA, El-Remessy AE, Al-Shabrawey $M$, Platt DH, Liou GI, Caldwell RW.

Trop J Pharm Res, February 2018; 17(2): 250 
Vascular endothelial growth factor and diabetic retinopathy: role of oxidative stress. Curr Drug Targets 2005; 6: 511-524.

26. Kanwar M, Chan PS, Kern TS, Kowluru RA. Oxidative damage in the retinal mitochondria of diabetic mice: possible protection by superoxide dismutase. Invest Ophthalmol Vis Sci 2007; 48: 3805-3811.

27. Nuñez G, Benedict MA, Hu Y, Inohara N. Caspases: the proteases of the apoptotic pathway. Oncogene 1998; 17: 3237-3245.

28. Zhao L, Yan X, Shi J, Ren F, Liu L, Sun S, Shan B. Ethanol extract of Forsythia suspensa root induces apoptosis of esophageal carcinoma cells via the mitochondrial apoptotic pathway. Mol Med Rep 2015; 11: $871-880$

29. Watanabe J, Nakamachi T, Ohtaki H, Naganuma A, Shioda S, Nakajo S. Low dose of methylmercury $(\mathrm{MeHg})$ exposure induces caspase mediated-apoptosis in cultured neural progenitor cells. J Toxicol Sci 2013; 38: 931-935.

30. O'Rourke B. Mitochondrial ion channels. Annu Rev Physiol 2007; 69: 19-49.

31. Feissner RF, Skalska J, Gaum WE, Sheu SS. Crosstalk signaling between mitochondrial $\mathrm{Ca} 2+$ and ROS. Front Biosci (Landmark Ed) 2009; 14: 1197-1218. 